\title{
Usefulness of the Pediatric Appendicitis Score and Neutrophil to Lymphocyte Ratio for Assessing the Complicated Appendicitis in Children
}

\section{Erdenetsetseg Chuluun1,2, Bayartsetseg Ankhbayar', Ganbayar Ganzorig², Ganbayar Luuzan ${ }^{3}$, Davaalkham Dambadarjaa4 ${ }^{4}$, Zorig Dungerdorj', Puntsag Chimedtseye ${ }^{1}$}

\author{
${ }^{1}$ Department of Surgery, School of Medicine, Mongolian National University of Medical Sciences, Ulaanbaatar, Mongolia \\ ${ }^{2}$ Mongolian-Japan Teaching Hospital, Mongolian National University of Medical Sciences, Ulaanbaatar, Mongolia \\ ${ }^{3}$ Mongolian National Center for Maternal and Child Health, Ulaanbaatar, Mongolia \\ ${ }^{4}$ School of Public Health, Mongolian National University of Medical Sciences, Ulaanbaatar, Mongolia \\ Email: Erdenetsetsegchuluun@gmail.com
}

How to cite this paper: Chuluun, E., Ankhbayar, B., Ganzorig, G., Luuzan, G., Dambadarjaa, D., Dungerdorj, Z. and Chimedtseye, P. (2020) Usefulness of the Pediatric Appendicitis Score and Neutrophil to Lymphocyte Ratio for Assessing the Complicated Appendicitis in Children. Open Journal of Clinical Diagnostics, 10, 93-103. https://doi.org/10.4236/ojcd.2020.104008

Received: November 25, 2020

Accepted: December 27, 2020

Published: December 30, 2020

Copyright $\odot 2020$ by author(s) and Scientific Research Publishing Inc. This work is licensed under the Creative Commons Attribution International License (CC BY 4.0).

http://creativecommons.org/licenses/by/4.0/

\section{(c) (i) Open Access}

\begin{abstract}
Introduction: Acute appendicitis (AA) is a common surgical disease which occurs in almost all age groups, and especially in childhood. Acute appendicitis is one of the most common causes of acute abdomen. The lifetime occurrence of this disease is approximately $7 \%$, with perforation rate of up to $20 \%$. In spite of the well-known classical symptoms and clinical findings of acute appendicitis, early diagnosis can be sometimes challenging. For the treatment of simple appendicitis (SA) in children, the effectiveness of antibiotic treatment has been reported. We aimed to determine predictive value of combination NLR and PAS in pediatric patients with clinical suspicion of acute appendicitis and complicated appendicitis Methods: Our study was performed on 480 children admitted for suspected acute appendicitis and underwent appendectomy at the MNCMCH, Ulaanbaatar Mongolia, between May 2019 and December 2019. White blood count (WBC), Neutrophil, NLR and PAS were compared between groups. Results: The sensitivity, specificity, PPV, NPV of PAS + NLR for differentiating complicated and noncomplicated appendicitis were $86.8 \%, 89.4 \%, 92.1 \%$ and $76 \%$ respectively. The sensitivity, specificity, PPV, NPV of PAS + NLR for diagnosis of acute appendicitis were $90.5 \%, 68.1 \%, 97.68 \%$ and $32.6 \%$ respectively. Conclusion: In the era of conservative antibiotic-based management of uncomplicated acute appendicitis, we advocate that combination of NLR and PAS is a useful aid in predicting complicated appendicitis.
\end{abstract}




\section{Keywords}

Acute Appendicitis, Complicated Appendicitis, Neutrophil to Lymphocyte Ratio, Antibiotic, Pediatric Patient

\section{Introduction}

Acute appendicitis (AA) is a common surgical disease which occurs in almost all age groups, and especially in childhood. The mortality is quite high especially when the diagnosis is late, and the rate of late diagnosis of acute appendicitis in children is also quite high between $30 \%$ and $65 \%$ [1]. Acute appendicitis is one of the most common causes of acute abdomen. The lifetime occurrence of this disease is approximately $7 \%$, with perforation rate of up to $20 \%$ [2]. In spite of the well-known classical symptoms and clinical findings of acute appendicitis, early diagnosis can be sometimes challenging. For the treatment of simple appendicitis (SA) in children, the effectiveness of antibiotic treatment has been reported. In appendicitis diagnosis, history and physical examination findings are important. The Pediatric Appendicitis Score (PAS) is used to diagnose acute appendicitis in children. The PAS is composed of simple items consisting of clinical symptoms, physical findings, and blood test findings. Since the PAS can be easily evaluated, it has been widely used. In addition to this, several blood tests are being used to predict appendicitis and its severity. While blood cells (WBC) counts are mostly elevated in patients with appendicitis [3]; however, an elevated WBC count has no predictive value in differentiating simple and complicated appendicitis [4] with the increase in number of tests, the cost is also increasing. For this reason, more simple and trustworthy parameters like Neutrophi/Lymphocyte $(\mathrm{N} / \mathrm{L})$ ratio are necessary. NLR provides information regarding two different immune and inflammatory pathways which may make it a potential marker to predict appendicitis and its severity. Neutrophil to lymphocyte ratio (NLR) is directly derived from the WBC, and its diagnostic relevance in AA was studied more in adults, although AA is more common in children. We aimed to determine predictive value of combination NLR and PAS in pediatric patients with clinical suspicion of acute appendicitis and complicated appendicitis. Our primary objective was to investigate whether NLR and PAS can predict acute appendicitis and whether it can distinguish between uncomplicated and complicated appendicitis. Our second objective was to determine cut-off values of NLR and PAS for appendicitis and complicated appendicitis.

\section{Methods}

\subsection{Subject}

Our study was performed on 480 children admitted for suspected acute appendicitis and underwent appendectomy at the MNCMCH, Ulaanbaatar Mongolia, between May 2019 and December 2019. Data were retrospectively collected from 
patients' medical records, including age, gender, symptoms and clinical signs, laboratory and histopathological findings as reference standard. The diagnosis of acute appendicitis in our hospital was established according to clinical manifestation, physical examination, laboratory findings and/or abdominal ultrasound. The final diagnosis was determined by histopathological finding.

\section{Exclusion criteria:}

- Incomplete medical records.

- Hematological disease, cancer.

- Poor speaking skills.

- Undergoing abdominal surgery.

- Chronic disease.

Inclusion criteria:

- Age of $<18$ years old.

- Underwent appendectomy.

- Abdominal organ anomalism.

- No hematological disease, cancer.

\subsection{Neutrophil Lymphocyte Ratio}

Laboratory data were collected including WBC, neutrophil and lymphocyte values. NLR was determined by dividing the neutrophil and lymphocyte values obtained.

\subsection{Pediatric Appendicitis Score}

\begin{tabular}{ccc}
\hline $\mathbf{N}^{\bullet}$ & PAS & Point \\
\hline $\mathbf{1}$ & Migration of pain & 1 \\
$\mathbf{2}$ & Anorexia & 1 \\
$\mathbf{3}$ & Nausea and vomiting & 1 \\
$\mathbf{4}$ & Elevated temperature $>38^{\circ} \mathrm{C}$ & 1 \\
$\mathbf{5}$ & Right lower quadrant tenderness & 2 \\
$\mathbf{6}$ & Cough, hopping, percussion tenderness in the right lower quadrant & 2 \\
7 & Increased leukocytes count & 1 \\
$\mathbf{8}$ & Neutrophilia & 1 \\
& Total & 10
\end{tabular}

\subsection{Statistical Analysis}

t-test was used to analyze the differences between groups when the data was normally distributed, while Mann Whitney U test was utilized when the data was not normally distributed. By comparing the means of two independent samples, the power of this study was 1.00 . The WBC, neutrophil and NRL were evaluated for sensitivity, specificity, positive predictive value (PPV), negative predictive value (NPV). The cutoff value of WBC, neutrophil, NLR and PAS for diagnosis of acute appendicitis and distinguishing noncomplicated appendicitis from the 
complicated ones were determined by receiver operating characteristics (ROC) curves.

\section{Result}

\subsection{Baseline Characteristics}

Most acute appendicitis patients in our institution were between the ages of 5 10 years old (43.75\%), male (56.05\%) and underwent laparotomy (0\%). The mean WBC and neutrophil count were $14.1 \pm 6 \times 10^{3} / \mu \mathrm{l}$ and $11.09 \pm 6.08$, respectively. Histopathological findings included complicated appendicitis in $76.07 \%$ of the children (Table 1).

\subsection{Accuracy of WBC, Neutrophil, Neutrophil-Lymphocyte Ratio and PAS for Diagnosis of Acute Appendicitis}

Neutrophil, NLR and WBC were significantly higher in the acute appendicitis group than control $(12.1 \pm 6.1$ vs. $7.8 \pm 4.4, \mathrm{p}<0.001$; and $9.25 \pm 6.1$ vs. $3.6 \pm$ $2.2, \mathrm{p}<0.001,14.72 \pm 6.02$ vs $12.3 \pm 5.5 \mathrm{p}<0.001$ respectively) (Table 2 ). The sensitivity, specificity, PPV, NPV, area under the ROC curve, and cutoff point of NLR, neutrophil and WBC for diagnosis of acute appendicitis were 89.4\%, $83.3 \%, 94.1 \%, 72.4 \%, 0.934$, and $4.97,62.5 \%, 75.8 \%, 88.5 \%, 40.2 \%, 0.716$ and 9.6 , $57.7 \%, 62.5 \%, 82.2 \%, 33.03 \%, 0.614$ and 12.7 respectively (Figure 1, Table 3). The sensitivity, specificity, PPV, NPV, and cutoff point of PAS for diagnosis of acute appendicitis were 90.8\%, 81.6\%, 93.6\%, 74.8\%, 6 respectively (Figure 2 (a), Table 4).

\subsection{Accuracy of WBC, Neutrophil, Neutrophil-Lymphocyte Ratio and PAS for Diagnosing CA}

WBC, neutrophil, and NLR were significantly greater in complicated than noncomplicated appendicitis $\left(15.3 \pm 5.94\right.$ vs. $13.6 \pm 6.04 \times 10^{3} / \mu \mathrm{L}, \mathrm{p}=0,008 ; 12,83$ \pm 6.12 vs. $7.79 \pm 4.46, \mathrm{p}<0.001$; and $12.06 \pm 6.57$ vs. $5.57 \pm 5.98, \mathrm{p}<0.001$, respectively) (Table 3). The sensitivity, specificity, PPV, NPV, area under the ROC curve, and cutoff point of NLR, neutrophil, and WBC for differentiating complicated and simple appendicitis were $81.9 \%, 85.24 \%, 91.5 \%, 70.74 \%, 0.899$, and 6.2 ; 63.6\%, 77.5\%, $89.65 \%, 41.5 \%, 0.751$, and 8.6 ; and 62.8\%, 75.4\%, 79.1\%, $32.3 \%, 0.589$ and 13.4 respectively (Figure 1, Table 2). The sensitivity, specificity, PPV, NPV, and cutoff point of PAS for differentiating complicated and simple appendicitis were $56.7 \%, 79.1 \%, 82.9 \%, 52.3 \%, 8$ respectively (Figure 2(b), Table 5).

\subsection{Accuracy of Combined of PAS and Neutrophil-Lymphocyte Ratio Distinguishing Noncomplicated and Complicated Appendicitis with Diagnosis of Acute Appendicitis}

The sensitivity, specificity, PPV, NPV of PAS + NLR for differentiating complicated and noncomplicated appendicitis were $86.8 \%, 89.4 \%, 92.1 \%$ and $76 \%$ respectively. The sensitivity, specificity, PPV, NPV of PAS + NLR for diagnosis of 
Table 1. Baseline characteristics of children with acute appendicitis.

\begin{tabular}{|c|c|}
\hline Characteristics & Mean \pm SD (min-max) \\
\hline \multicolumn{2}{|l|}{ Age } \\
\hline$<5$ & $25(5.2 \%)$ \\
\hline $5-10$ & $210(43.75 \%)$ \\
\hline $10<$ & $245(51.04 \%)$ \\
\hline \multicolumn{2}{|l|}{ Gender } \\
\hline Female & $211(43.95 \%)$ \\
\hline Male & $269(56.05 \%)$ \\
\hline WBC & $14.1 \pm 6(3.5-30.43)$ \\
\hline Neutrophil & $11.09 \pm 6.08(1.07-19.67)$ \\
\hline \multicolumn{2}{|l|}{ Clinical characteristics } \\
\hline Elevated temperature $>38^{\circ} \mathrm{C}$ & $182(37.9 \%)$ \\
\hline Cough, hopping, percussion tenderness in the right lower quadrant & $269(56 \%)$ \\
\hline Right lower quadrant tenderness & $394(82.1 \%)$ \\
\hline Nausea and vomiting & $320(66.6 \%)$ \\
\hline Anorexia & $327(68.1 \%)$ \\
\hline Migration of pain & $242(50.4 \%)$ \\
\hline Increased leukocytes count & $351(73.1 \%)$ \\
\hline Neutrophilia & $301(62.7 \%)$ \\
\hline \multicolumn{2}{|l|}{ Surgical Approach } \\
\hline Appendectomy & $480(100 \%)$ \\
\hline Laparoscopic & $0(0 \%)$ \\
\hline \multicolumn{2}{|l|}{ Histopathological Findings } \\
\hline Noncomplicated & $86(23.83 \%)$ \\
\hline Complicated & $275(76.17 \%)$ \\
\hline
\end{tabular}

Table 2. The accuracy of WBC, neutrophil and NLR for diagnosis of complicated acute appendicitis in children.

\begin{tabular}{cccccccccc}
\hline Mean & Complicated & Noncomplicated & p value & AUC & Cut-off level & Sen & Spe & PPV & NPV \\
\hline NLR & $12.06(6.57)$ & $5.57(5.98)$ & $0.001>$ & 0.899 & 6.2 & $81.9 \%$ & $85.24 \%$ & $91.5 \%$ & $70.74 \%$ \\
WBC & $15.3(5.94)$ & $13.6(6.04)$ & 0.008 & 0.589 & 13.4 & $62, .8 \%$ & $75.4 \%$ & $79.1 \%$ & $32.3 \%$ \\
Neutrophil & $12.83(6.12)$ & $7.79(4.46)$ & $0.001>$ & 0.751 & 8,6 & $63.6 \%$ & $77.5 \%$ & $89.65 \%$ & $41.5 \%$ \\
\hline
\end{tabular}

Table 3. The accuracy of WBC, neutrophil and NLR for diagnosis of acute appendicitis in children.

\begin{tabular}{cccccccccc}
\hline Mean & Positive appendectomy & Negative appendectomy & p value & AUC & Cut-offlevel & Sen & Spe & PPV & NPV \\
\hline NLR & $9.25 \pm 6.1$ & $3.6 \pm 2.2$ & $0.001>$ & 0.934 & 4.97 & $89.4 \%$ & $83.3 \%$ & $94.1 \%$ & $72.4 \%$ \\
WBC & $14.72 \pm 6.02$ & $12.3 \pm 5.5$ & $0.001>$ & 0.614 & 12.7 & $57.7 \%$ & $62.5 \%$ & $82.2 \%$ & $33.03 \%$ \\
Neutrophil & $12.1 \pm 6.1$ & $7.8 \pm 4.4$ & $0.001>$ & 0.716 & 9.6 & $62.5 \%$ & $75.8 \%$ & $88.5 \%$ & $40.2 \%$ \\
\hline
\end{tabular}




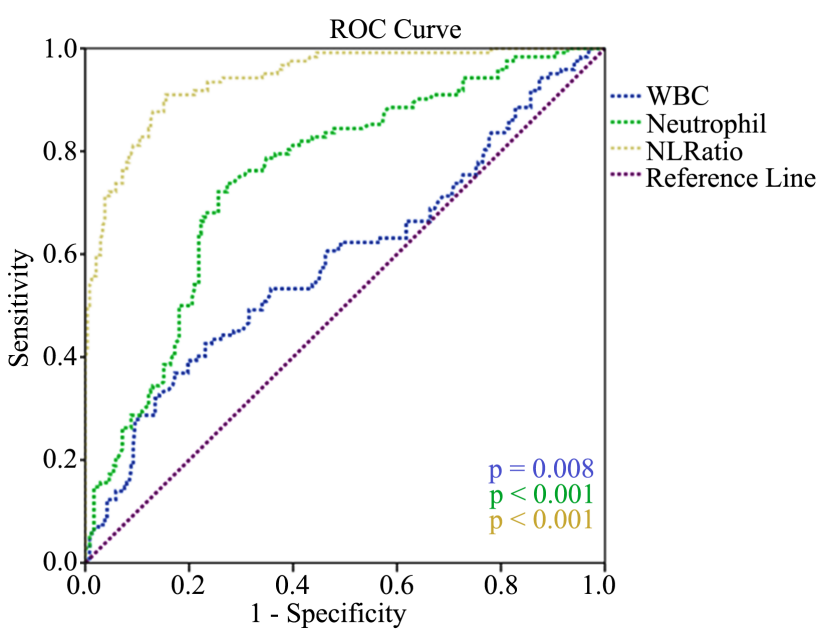

(a)

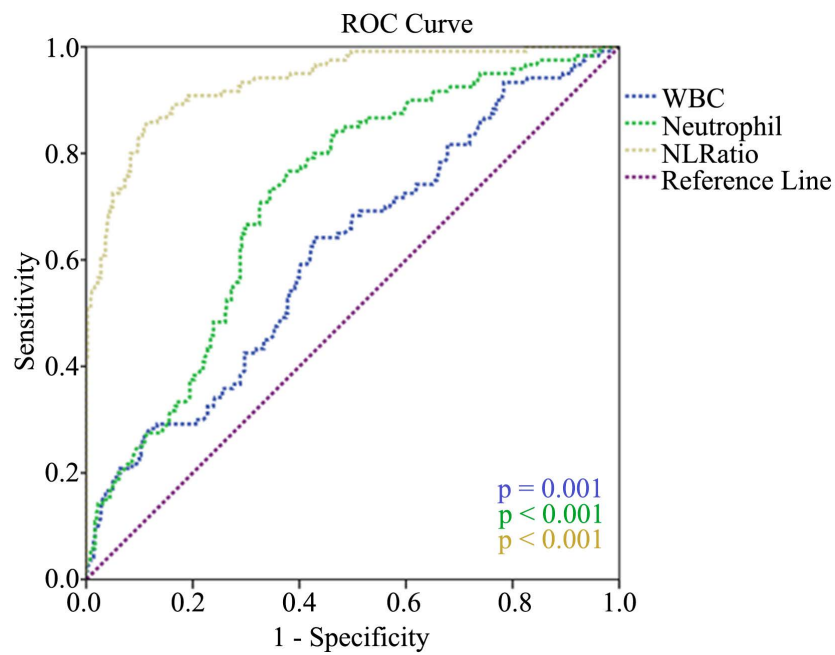

(b)

Figure 1. (a) ROC curve of WBC, Neutrophil, and NLR for diagnosing CA; (b) ROC curve of WBC, Neutrophil and NLR for diagnosis of acute appendicitis.

Table 4. Sensitivity, specificity, PPV, and NPV of the PAS for diagnosing appendicitis.

\begin{tabular}{ccccc}
\hline PAS & Sensitivity & Specificity & PPV & NPV \\
PAS $\geq 1$ & $100 \%$ & $0 \%$ & $75 \%$ & $0 \%$ \\
PAS $\geq 2$ & $99.7 \%$ & $7.5 \%$ & $76.38 \%$ & $90 \%$ \\
PAS $\geq 3$ & $99.7 \%$ & $17.5 \%$ & $76.38 \%$ & $90 \%$ \\
PAS $\geq 4$ & $99.1 \%$ & $27.5 \%$ & $80.4 \%$ & $91.6 \%$ \\
PAS $\geq 5$ & $51.6 \%$ & $85.7 \%$ & $83.78 \%$ \\
PAS $\geq 6$ & $96.6 \%$ & $81.6 \%$ & $93.6 \%$ & $74.8 \%$ \\
PAS $\geq 7$ & $90.8 \%$ & $87.5 \%$ & $93.6 \%$ & $43.03 \%$ \\
PAS $\geq 8$ & $61.38 \%$ & $96.6 \%$ & $97.1 \%$ & $34.11 \%$ \\
PAS $\geq 9$ & $37.7 \%$ & $99.1 \%$ & $97.9 \%$ & $72.38 \%$ \\
PAS $=10$ & $13.3 \%$ & $100 \%$ & $100 \%$ & $25.2 \%$ \\
\hline
\end{tabular}




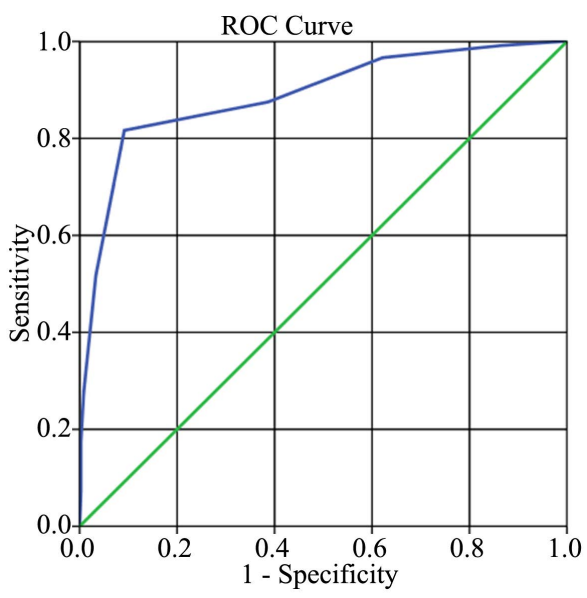

(a)

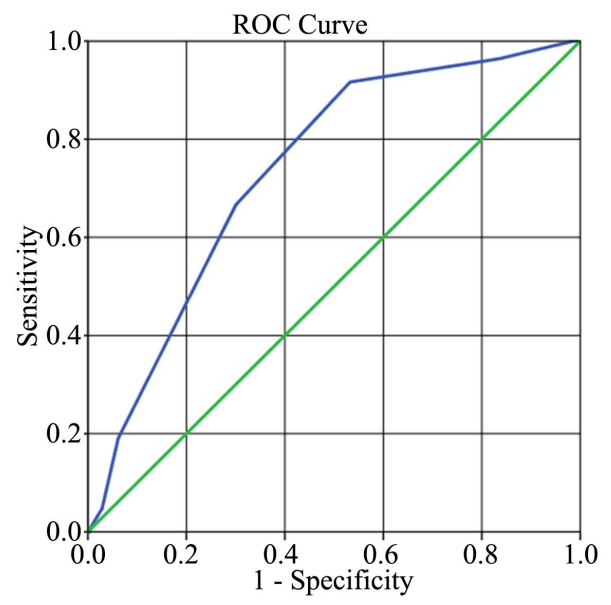

(b)

Figure 2. (a) Receiver operating characteristic curve of PAS for diagnosing acute appendicitis; (b) Receiver operating characteristic curve of PAS for diagnosing CA.

Table 5. Sensitivity, specificity, PPV, and NPV of the PAS for diagnosing CA.

\begin{tabular}{ccccc}
\hline PAS & Sensitivity & Specificity & PPV & NPV \\
\hline PAS $\geq 1$ & $100 \%$ & $0 \%$ & $76.6 \%$ & $0 \%$ \\
PAS $\geq 2$ & $99.6 \%$ & $0 \%$ & $76.3 \%$ & $0 \%$ \\
PAS $\geq 3$ & $99.6 \%$ & $0 \%$ & $76.3 \%$ & $0 \%$ \\
PAS $\geq 4$ & $99.27 \%$ & $1.1 \%$ & $76.7 \%$ & $33.3 \%$ \\
PAS $\geq 5$ & $97.1 \%$ & $4.7 \%$ & $77.0 \%$ & $33.3 \%$ \\
PAS $\geq 6$ & $93.8 \%$ & $19.04 \%$ & $79.2 \%$ & $48.48 \%$ \\
PAS $\geq 7$ & $69.9 \%$ & $66.6 \%$ & $87.3 \%$ & $40.28 \%$ \\
PAS $\geq \mathbf{8}$ & $46.7 \%$ & $\mathbf{8 1 . 6 \%}$ & $\mathbf{8 8 . 8} \%$ & $34.37 \%$ \\
PAS $\geq 9$ & $16.3 \%$ & $96.4 \%$ & $93.75 \%$ & $25.96 \%$ \\
PAS $=\mathbf{1 0}$ & $1.4 \%$ & $100 \%$ & $100 \%$ & $23.59 \%$ \\
\hline
\end{tabular}

acute appendicitis were 90.5\%, 68.1\%, 97.68\% and 32.6\% respectively (Table 6 and Table 7). 
Table 6. Diagnostic accuracy of PAS, NLR and PAS + NLR for CA.

\begin{tabular}{cccc}
\hline & PAS & NLR & PAS + NLR \\
\hline Sensitivity & $46.7 \%$ & $81.9 \%$ & $86.8 \%$ \\
Specificity & $81.6 \%$ & $85.24 \%$ & $89.4 \%$ \\
PPV & $88.8 \%$ & $91.5 \%$ & $92.1 \%$ \\
NPV & $34.37 \%$ & $70.74 \%$ & $76 \%$ \\
\hline
\end{tabular}

Table 7. Diagnostic accuracy of PAS, NLR and PAS + NLR for acute appendicitis.

\begin{tabular}{cccc}
\hline & PAS & NLR & PAS + NLR \\
\hline Sensitivity & $90.8 \%$ & $89.4 \%$ & $90.5 \%$ \\
Specificity & $81.6 \%$ & $83.3 \%$ & $68.1 \%$ \\
PPV & $93.6 \%$ & $94.1 \%$ & $97.68 \%$ \\
NPV & $74.8 \%$ & $72.4 \%$ & $32.6 \%$ \\
\hline
\end{tabular}

\section{Discussion}

For the treatment of SA in children, the effectiveness of antibiotic treatment has been reported [5] [6]. A meta-analysis showed that initial antibiotic treatment of SA was comparable with appendectomy, with a high rate of success, and treatment with antibiotics alone was not associated with increased complications [5]. Thus, accurate distinction between SA and CA is important, as antibiotic treatment for SA could be an option as initial treatment. Non-operative management with antibiotics alone may be an option for patients with uncomplicated appendicitis; however, surgery is still the first choice for complicated AA, and especially gangrenous appendicitis. The PAS is mainly scored based on clinical symptoms, physical findings, and differential WBC. Because of its convenience, this score has been widely used as a diagnostic tool of acute appendicitis in children. A score $\geq 6$ was reported compatible with the diagnosis of appendicitis. However, there has been no report on how many points of the PAS are likely to indicate CA. We found that there was a statistically significant difference in the PAS between SA and CA. Thus, the PAS may be correlated with histopathological progression. In addition, the Youden index cut-off value of the PAS for diagnosing CA was 8 . At 8 points, the AUC was 0.793 . This value was recognized as a good value of accuracy for diagnosing CA. Additionally, the PPV of a PAS $\geq$ 8 for diagnosing CA was $88.8 \%$, which was reasonable for diagnostic use. We are able to show that NLR has a high accuracy to diagnose acute appendicitis in children with sensitivity of $\sim 0.84$. Our finding is similar with previous studies that showed NLR is a good parameter for diagnosis of acute appendicitis [7] [8] although with different cutoff value (our study: 4.97 vs 2.87 [9] vs. 3.5 [7]). Furthermore, our patients do not show any abnormality in their appendix $(20.42 \%)$ and some even have a non-visualized appendix (47.08\%) during abdominal ultrasound. It has been known that ultrasound results are very dependent on the operator and have low accuracy for diagnosis of acute appendicitis. A study conducted by Yazici et al. showed that a NLR over 3.5 in a pediatric patient 
group had maximum sensitivity and more sensitivity than the WBC count. Another study by Ishizuka et al. revealed that an NLR $>8$ had a significant association with gangrenous appendicitis in patients undergoing appendectomy. Markar et al. assessed 1117 pediatric patients who underwent appendectomy and determined that the NLR appeared to be of greater diagnostic accuracy than the total WBC count. According to the present results, WCC is a significant parameter for the diagnosis of acute appendicitis. However, it is not a perfect indicator, due to relatively low sensitivity and specificity. With a cut-off value of $11,900 / \mathrm{mm}^{3}, 71 \%$ sensitivity and $68 \%$ specificity were found. In a recent study, Rafiq et al. [10] reported very high sensitivity and specificity ( $87 \%$ and $92 \%$, respectively) with the same cut-off value. Nevertheless, in previous studies, sensitivity and specificity of WCC have been reported between $67 \%-87 \%$ and $43 \%$ $81 \%$, respectively. The argument that NLR is a more sensitive parameter than the number of leukocytes was put forth 20 years earlier by Goodman et al. [11] Four studies have been published in the last 5 years regarding this issue. Shimizu et al. [12] suggest a NLR cut-off value of 5.0 for the diagnosis of acute appendicitis, with $44 \%$ sensitivity and $22 \%$ specificity. Ishizuka et al. [13] determined a cut-off value of 8.0 for NLR to differentiate gangrenous appendicitis from catarrhal appendicitis, with $73 \%$ sensitivity and $39 \%$ specificity. Kahramanca et al. [14] reported 2 NLR cut-off values of 4.68 (65\% sensitivity, 55\% specificity) and 5.74 (71\% sensitivity, $49 \%$ specificity) to distinguish acute appendicitis from normal appendix, and complicated appendicitis from non-complicated appendicitis, respectively. Sevinç et al. NLR cut-off values were 3.0 (81\% sensitivity, 53\% specificity) and 5.5 (78.4\% sensitivity, $41.7 \%$ specificity) for the diagnosis of acute appendicitis and perforated appendicitis, respectively. Khairol et al. reported the cut-off point of NLR in this study showed a highly statistically significant difference in an intergroup comparison. It showed that NLR with a cut-off point of $\geq 3.11$ could significantly differentiate normal appendix and acute appendicitis patients with a sensitivity of $75.2 \%$ and specificity of $68.7 \%$, in diagnosing acute appendicitis. Moreover, most of our patients are male $(56.05 \%)$ with an average age of 5 - 10 years (43.75\%). These findings were compatible with previous study [15]. Acute appendicitis is more commonly found in adolescents, which might be associated with the large proportion of lymphoid tissue during adolescence, resulting in the appendix being susceptible to obstruction and inflammation.

\section{Conclusion}

In the era of conservative antibiotic-based management of simple uncomplicated acute appendicitis, we advocate that combination of NLR and PAS is a useful aid in predicting complicated appendicitis.

\section{Conflicts of Interest}

The authors declare no conflicts of interest regarding the publication of this paper. 


\section{References}

[1] Humes, D. and Simpson, J. (2006) Acute Appendicitis. BMJ, 333, 530-534. https://doi.org/10.1136/bmj.38940.664363.AE

[2] Storm-Dickerson, T.L. and Horattas, M.C. (2003) What Have We Learned over the Past 20 Years about Appendicitis in the Elderly? The American Journal of Surgery, 185, 198-201. https://doi.org/10.1016/S0002-9610(02)01390-9

[3] Guraya, S.Y., Al-Tuwaijri, T.A., Khairy, G.A. and Murshid, K.R. (2005) Validity of Leukocyte Count to Predict the Severity of Acute Appendicitis. Saudi Medical Journal, 26, 1945-1947.

[4] Sand, M., Bechara, F.G., Holland-Letz, T., Sand, D., Mehnert, G. and Mann, B. (2009) Diagnostic Value of Hyperbilirubinemia as a Predictive Factor for Appendiceal Perforation in Acute Appendicitis. The American Journal of Surgery, 198, 193-198. https://doi.org/10.1016/j.amjsurg.2008.08.026

[5] Huang, L.B., Yin, Y., Yang, L., Wang, C., Li, Y. and Zhou, Z.G. (2017) Comparison of Antibiotic Therapy and Appendectomy for Acute Uncomplicated Appendicitis in Children: A Meta-Analysis. JAMA Pediatrics, 171, 426-434. https://doi.org/10.1001/jamapediatrics.2017.0057

[6] Gorter, R.R., The, S.-M.M., Gorter-Stam, M.A., Eker, H.H., Bakx, R., van der Lee, J.H. and Heij, H.A. (2017) Systematic Review of Nonoperative versus Operative Treatment of Uncomplicated Appendicitis. Journal of Pediatric Surgery, 52, 1219-1227. https://doi.org/10.1016/j.jpedsurg.2017.04.005

[7] Yazici, M., Özkisacik, S., Öztan, M.O. and Gürsoy, H. (2010) Neutrophil/Lymphocyte Ratio in the Diagnosis of Childhood Appendicitis. The Turkish Journal of Pediatrics, 52, 400-403.

[8] Markar, S., Karthikesalingam, A., Falzon, A. and Kan, Y. (2010) The Diagnostic Value of Neutrophil: Lymphocyte Ratio in Adults with Suspected Acute Appendicitis. Acta Chirurgica Belgica, 110, 543-547. https://doi.org/10.1080/00015458.2010.11680673

[9] Prasetya, D. (2019) Accuracy of Neutrophil Lymphocyte Ratio for Diagnosis of Acute Appendicitis in Children: A Diagnostic Study. Annals of Medicine and Surgery, 48, 35-38. https://doi.org/10.1016/j.amsu.2019.10.013

[10] Rafiq, M.S., Khan, M.M., Khan, A. and Ahmad, B. (2015) Total Leukocyte and Neutrophil Count as Preventive Tools in Reducing Negative Appendectomies. Turkish Journal of Trauma and Emergency Surgery, 21, 102-106. https://doi.org/10.5505/tjtes.2015.29626

[11] Goodman, D.A. and Goodman, C.B. (1995) Use of the Neutrophil: Lymphocyte Ratio in the Diagnosis of Appendicitis. American Surgeon, 61, 257-259.

[12] Shimizu, T., Ishizuka, M. and Kubota, K. (2016) A Lower Neutrophil to Lymphocyte Ratio Is Closely Associated with Catarrhal Appendicitis versus Severe Appendicitis. Surgery Today, 46, 84-89. https://doi.org/10.1007/s00595-015-1125-3

[13] Ishizuka, M., Shimizu, T. and Kubota, K. (2013) Neutrophil-to-Lymphocyte Ratio Has a Close Association with Gangrenous Appendicitis in Patients Undergoing Appendectomy. International Surgery, 97, 299-304. https://doi.org/10.9738/CC161.1

[14] Kahramanca, Ş., Özgehan, G., Şeker, D., Gökce, E.İ., Şeker, G., Tunç, G., Küçükpınar, T. and Kargıc1, H. (2014) Neutrophil-to-Lymphocyte Ratio as a Predictor of Acute Appendicitis. Turkish Journal of Trauma and Emergency Surgery, 20, 19-22. https://doi.org/10.5505/tjtes.2014.20688 
[15] Bhangu, A., Søreide, K., Di Saverio, S., Assarsson, J.H. and Drake, F.T. (2015) Acute Appendicitis: Modern Understanding of Pathogenesis, Diagnosis, and Management. The Lancet, 386, 1278-1287. https://doi.org/10.1016/S0140-6736(15)00275-5 